\title{
RF TESTS OF AN 805 MHZ PILLBOX CAVITY AT LAB G OF FERMILAB*
}

\author{
Derun Li, J. Corlett, R. MacGill, J. Wallig, M. Zisman, LBNL, Berkeley, CA 94720, USA \\ A. Moretti, Z. Qian, V. Wu, FNAL, Batavia, IL, USA \\ R. Rimmer, Jefferson National Accelerator Facility, Newport News, VA, USA \\ J. Norem, ANL, Argonne, IL, USA \\ Y. Torun, Illinois Institute of Technology, Chicago, IL, USA
}

\begin{abstract}
We report recent high power RF tests on an $805 \mathrm{MHz}$ RF pillbox cavity with demountable windows over beam apertures at Lab G of Fermilab, a dedicated facility for testing of MUCOOL (muon cooling) components. The cavity is installed inside a superconducting solenoidal magnet. A $12 \mathrm{MW}$ peak RF power klystron is used for the tests. The cavity has been processed both with and without magnetic field. Without magnetic field, a gradient of $34 \mathrm{MV} / \mathrm{m}$ was reached rather quickly with very low sparking rate. In a $2.5 \mathrm{~T}$ solenoidal field, a $16 \mathrm{MV} / \mathrm{m}$ gradient was achieved, following several weeks of conditioning. Strong multipacting effects associated with high radiation levels were measured during processing with the magnetic field. More recently Be windows with TiN-coated surface have been installed and tested with and without the external magnetic field. $16 \mathrm{MV} / \mathrm{m}$ gradient without magnetic field was reached quickly as planned. Less multipacting was observed during the conditioning, indicating that the TiN-coated surface on the windows had indeed helped to reduce the secondary electron emission significantly. A gradient of $16.5 \mathrm{MV} / \mathrm{m}$ was finally achieved with magnet on in solenoidal mode and the field up to $4 \mathrm{~T}$. Preliminary inspection of the Be window surface found no visual damage, in comparison with $\mathrm{Cu}$ windows where substantial surface damage was found. Preliminary understanding of conditioning the cavity in a strong magnetic field has been developed. More thorough window and cavity surface inspection is under way.
\end{abstract}

\section{INTRODUCTION}

Accelerators for intense muon beams call for very high gradient RF cavities at various frequencies. The high gradient is required to manipulate muon beams that are created with a large 6-D phase space, and decay rapidly (muon lifetime is about $2 \mu \mathrm{s}$ at rest). Any manipulation to the muon beams has to be done quickly, including cooling. For instance, on the muon cooling channel design for Study-II [1] calls for accelerating gradient of $\sim 16$ $\mathrm{MV} / \mathrm{m}$ at $201 \mathrm{MHz}$, compared to $\sim 15 \mathrm{MV} / \mathrm{m}$ Kilpatrick criterion at the same frequency. Moreover these cavities have to be installed inside a superconducting magnet where magnetic fields are as high as a few Tesla. Therefore normal (warm) conducting RF cavity technology has to be used. Cavity geometry optimization alone is not sufficient to reduce the peak surface field and at the same time provide high shunt impedance with the large beam iris needed for muon beams. Taking advantage of the muon penetration property, a closed cell RF cavity was proposed [2] where the large beam iris may be terminated by thin low $\mathrm{Z}$ conducting material. The closed cell cavity resembles a cylindrical pillbox cavity where peak surface field is the same as the peak acceleration field on beam axis. Furthermore such a cavity design allows for arbitrary choices of phase advance per cavity, independent phase control of each cavity, and more freedom for beam dynamics design. Efforts to design such a high gradient cavity have highlighted the engineering challenges on how to incorporate thin $\mathrm{Be}$ windows in the cavity. Conditioning and operating a RF cavity in strong magnetic field of a few-T pose potential difficulties in overcoming multipacting zones, surface damage and many possible unknowns. As part of ongoing $\mathrm{R} \& \mathrm{D}$ activity for the high gradient RF cavity for muon beams, we have designed and constructed an $805 \mathrm{MHz}$ pillbox cavity with demountable windows [3]. The cavity design allows for tests of different windows. The cavity has been under high power RF tests at Lab G, FNAL since March 2002. In this paper, we report recent test results and future plans. Preliminary data analysis will be presented. Surface inspection and analysis of windows are being analyzed, and is reported in another paper at this conference [4].

\section{THE 805 MHZ CAVITY}

The $805 \mathrm{MHz}$ pillbox cavity was reported previously [3]. The cavity and its power coupler were designed at LBNL using the MAFIA code in frequency and time domain, respectively. Good agreement was achieved between the design values and low power RF measurements. The cavity body was fabricated at the University of Mississippi, with final cavity braze carried out at the Alpha-Braze Company in Fremont, California. Final cavity cleaning, assembly, system integration and vacuum checking were conducted at LBNL. Four prestressed flat $\mathrm{Be}$ windows were purchased from BrushWellman Company in Fremont for tests. The cavity was shipped to Lab G of Fermilab for high power tests in March 2002. Table 1 lists the main RF parameters of this cavity in comparison with the low power RF measurements at Lab G. 
Table 1: Main parameters of the cavity in comparison with low power microwave measurements

\begin{tabular}{|l|c|c|c|}
\hline Name & Design & Measured & Unit \\
\hline Frequency & 805.00 & 804.946 & $\mathrm{MHz}$ \\
\hline $\mathrm{Q}_{0}$ & 18,800 & 15,080 & \\
\hline Coupling constant & 1.0 & 1.08 & \\
\hline Radius & 15.62 & & $\mathrm{Cm}$ \\
\hline Gap Length & 8.1 & & $\mathrm{~cm}$ \\
\hline Window diameter & 16 & & \\
\hline Be window thickness & 0.127 & & $\mathrm{~mm}$ \\
\hline Gradient on axis & 30 & & $\mathrm{MV} / \mathrm{m}$ \\
\hline Shunt Impedance & 32 & & $\mathrm{M} \Omega / \mathrm{m}$ \\
\hline
\end{tabular}

\section{RF TEST OF THE CAVITY}

\section{Test Set Up at Lab G}

A $12 \mathrm{MW}$ peak power klystron and a superconducting solenoid magnet up to 5 Tesla are available for the RF tests. The SC magnet can be powered to operate in either solenoidal mode or gradient mode. Figure 1 is a photo taken inside Lab G test cave showing the test set up.

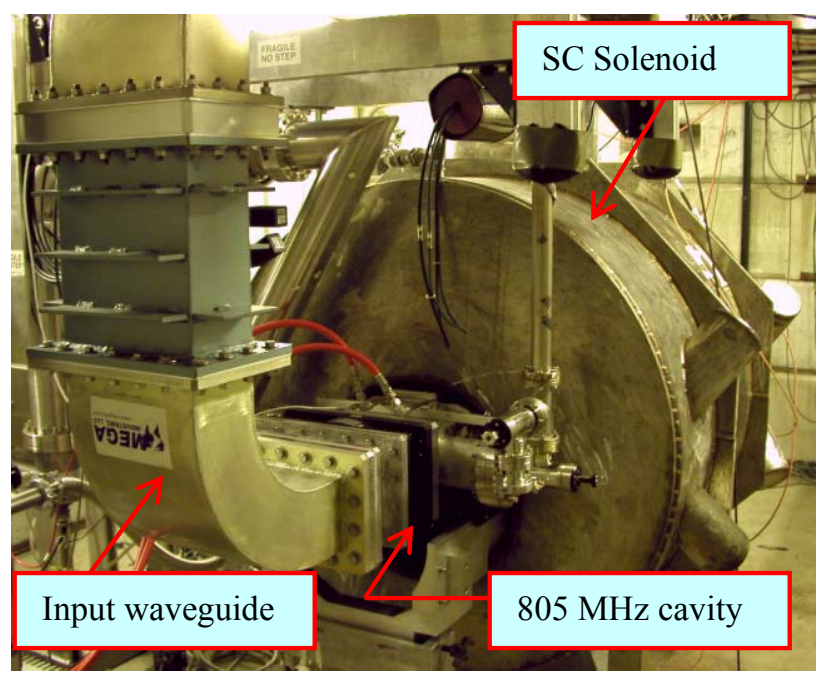

Figure 1: RF cavity inside the SC solenoid at Lab G, FNAL

The $805 \mathrm{MHz}$ pillbox cavity sits inside the center of the warm bore of the superconducting solenoid. All necessary measurement equipment and probes needed for RF, vacuum, $\mathrm{x}$-ray and dark current measurement are available. The RF tests can be set and controlled either by computers or manually.

\section{Test Results}

The cavity was first baked up to $115{ }^{\circ} \mathrm{C}$ with hot $\mathrm{N}_{2}$ purge for 2 days and then pumped down to vacuum of order of $10^{-8}$ Torr before RF conditioning started. We will report the test results according to the timeline. High power $\mathrm{RF}$ conditioning was conducted on $\mathrm{Cu}$ windows

\footnotetext{
* Shunt impedance definition used here: $\mathrm{ZT}^{2}=\mathrm{V}^{2} / \mathrm{P}$
}

first, and then followed tests of Be windows with one TiN-coated surface facing RF, with and without magnetic field.

\section{Test of Cu Window without External Magnetic Field}

High power RF conditioning started with $\mathrm{Cu}$ windows, rather than $\mathrm{Be}$ windows initially, without magnet the on. Combinations of different pulse lengths and repetition rates were used in conditioning phases. Multipacting effects were observed at low RF power as expected. Typical two-plate multipacting zones were observed, and passed rather quickly. $34 \mathrm{MV} / \mathrm{m}$ accelerating gradient was achieved with low x-rays, low dark current and little sparking. This gradient has exceeded our designed value of $30 \mathrm{MV} / \mathrm{m}$. The cavity was then taken apart for surface inspection on cavity body and windows, no surface damage was found.

\section{Test of $\mathrm{Cu}$ Window with External Magnetic Field}

The cavity tests resumed with the super-conducting magnet on at $2.5 \mathrm{~T}$ in solenoidal mode. The cavity seemed to have lost its conditioning history and behave like a brand new cavity again. More multipacting zones popped up, and they were much harder to pass through. Radiation levels went up by almost 1000 times, compared to the case without the external magnetic field. Details on x-ray, dark current and radiation measurements are reported in [4]. Higher radiation levels and more severe sparking prevented us from pursuing higher accelerating gradients. Nevertheless we found a possible surface self-healing effect that appeared during a period of the conditioning. That is, after a period of conditioning with the magnet on, associated with strong multipacting zones and high radiation levels, we turned the magnet off and continued re-conditioning the cavity without magnetic field for one or more days. The dark currents and radiation levels were measured to be dropping continuously with the conditioning time. This process was repeated and verified. Higher or equal gradient was then re-gained at much lower radiation levels. This suggests reconditioning without the external magnetic fields seem to be able to cure or condition the damaged surface caused with the strong magnetic field.

Experiments indicated that it is difficult to run an RF cavity in a strong magnetic field without extensive conditioning. We are still exploring the best way to condition a cavity in such an environment. In addition to RF fields in the cavity, the external magnetic field (in the longitudinal direction) forms a strong focusing channel for electrons and ions and forces them to orbit within the channel. As this focusing force from the external magnetic field is much stronger then that of RF field, multipacting zones are no longer bound in the region of the two parallel windows at low RF powers only. Multipacting conditions may be satisfied at higher powers and larger radius. Numerical simulations of experimental conditions are required and will be conducted in the near future to further study and understand these effects. 
A modest $18 \mathrm{MV} / \mathrm{m}$ accelerating gradient was reached with the $\mathrm{Cu}$ windows at $2.5 \mathrm{~T}$ magnetic field in solenoidal mode. The $\mathrm{Cu}$ windows were taken out for surface examination after reaching this gradient. Surface damage on the windows was apparent, as shown in Figure 2.
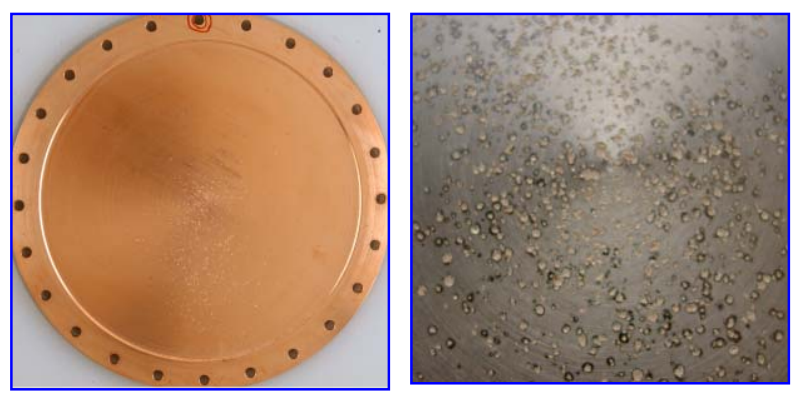

Figure 2: $\mathrm{Cu}$ window after RF conditioning with the external $2.5 \mathrm{~T}$ magnetic field in solenoidal mode. Surface damage (pitting) can be seen clearly on the photo (right), which is a close-up view of the center region of the same window.

\section{Test of Be Windows with TiN Coated Surface Without External Magnetic Field}

Two Be windows with TiN coated surface were installed face-to-face in the cavity. They are pre-stressed flat windows of 0.25 and $0.5 \mathrm{~mm}$ thickness, respectively. The coating thickness is $\sim 200 \mathrm{~A}$ which is intended to suppressing multipacting. As was experienced in previous tests of the $\mathrm{Cu}$ windows, conditioning with an external magnetic field results in window surface damage. A modest gradient of $16 \mathrm{MV} / \mathrm{m}$ was set for conditioning without magnetic field. The purpose of this test was to make sure that we can condition the cavity with the thin Be windows, and yet not damage the window surface before switching the magnet on. Less multipacting was indeed observed as expected from the TiN-coated surface. The target of $16 \mathrm{MV} / \mathrm{m}$ was achieved quickly without difficulty.

Small cavity frequency shifts were measured during the conditioning. This suggested that the pre-stressed thin $\mathrm{Be}$ windows may have deformed slightly (lost their tension) or vibrated due to either RF heating or the RF impulse. The frequency shifts were small and slow, and they were well within the bandwidth of the klystron. Conditioning was continued with manual adjustment of the drive frequency.

\section{Test of Be Windows with TiN-coated Surface With External Magnetic Field}

The high power tests were resumed with the TiN-coated Be windows with the solenoid magnet on. After weeks of conditioning, only $16.5 \mathrm{MV} / \mathrm{m}$ gradient was achieved with the magnetic field up to $4 \mathrm{~T}$. Higher than this gradient would result in more sparking. Fearing possible damage to the $\mathrm{Be}$ windows (Be is a health hazardous material), we stopped the test at this gradient and inspected the window surface. Fig. 3 shows a $\mathrm{Cu}$ and a $\mathrm{Be}$ window side by side after the conditioning, for comparison purpose.
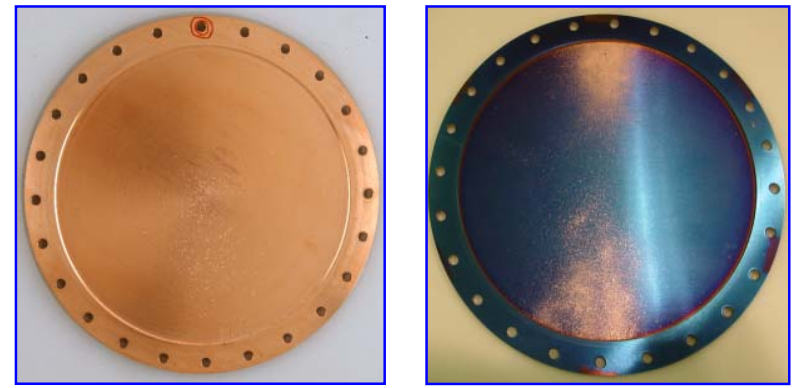

Figure 3: $\mathrm{Cu}$ window (left) and $\mathrm{Be}$ window (right) after $\mathrm{RF}$ conditioning with magnetic field. Deposited $\mathrm{Cu}$ particles on the Be window can clearly be seen.

Preliminary surface analysis indicated no surface damage to the Be window. Copper particles or beads were deposited on the Be window surface. This indicated that they must come from cavity body. Further inspection found that there was minor surface damage around the iris area where the $\mathrm{Be}$ windows were bolted on. The distribution pattern of the $\mathrm{Cu}$ particles (beads) is not well understood, and will be further studied by numerical simulations and experiments in near future.

\section{Future Test Plan}

A new pair of Be windows with TiN-coated surface have been installed and are ready for tests to the highest gradient. Bare Be windows will be tested for multipacting studies. Other window options such as pre-curved windows or grids may be designed and tested at Lab G.

\section{CONCLUSION}

We have tested the cavity with $\mathrm{Cu}$ and $\mathrm{Be}$ windows with and without external magnetic fields. $16.5 \mathrm{MV} / \mathrm{m}$ was achieved with TiN-coated Be windows with a $4 \mathrm{~T}$ magnet on. We believe the gradient is not limited by the $\mathrm{Be}$ windows. Preliminary surface analysis indicated no damage on the $\mathrm{Be}$ windows which further suggests that Be windows can withstand high RF gradient in a few- $\mathrm{T}$ magnetic field. Coating on high field region of the cavity body, say TiN, may reduce multipacting and improve the cavity performance.

\section{REFERENCES}

[1] M. M. Alsharo'a, et al., "Status of neutrino Factory and Muon Collider Research and Development and Future Plans", PRST-Accelerators \& Beams (2002)

[2] W. Turner, et al., "RF System Concept for a Muon Cooling Experiment", EPAC 1998, Sweden

[3] D. Li, et al, "High Power RF Test of an $805-\mathrm{MHz}$ Cavity for a Muon Cooling Channel", EPAC 2002, Paris, France

[4] J. Norem, et al., "Dark Current and X-ray Measurements of an $805 \mathrm{MHz}$ Pillbox Cavity", this conference. 